\title{
Decisional responsibility for mechanical ventilation and weaning: an international survey
}

Louise Rose ${ }^{1,2,3,4^{*}}$, Bronagh Blackwood ${ }^{5}$, Ingrid Egerod ${ }^{6}$, Hege Selnes Haugdahl ${ }^{7}$, José Hofhuis ${ }^{8}$, Michael Isfort ${ }^{9}$, Kalliopi Kydonaki ${ }^{10}$, Maria Schubert ${ }^{11,12}$, Riccardo Sperlinga ${ }^{13,14}$, Peter Spronk ${ }^{15}$, Sissel Storli ${ }^{16}$, Daniel F McAuley ${ }^{17}$ and Marcus J Schultz ${ }^{18}$

\begin{abstract}
Introduction: Optimal management of mechanical ventilation and weaning requires dynamic and collaborative decision making to minimize complications and avoid delays in the transition to extubation. In the absence of collaboration, ventilation decision making may be fragmented, inconsistent, and delayed. Our objective was to describe the professional group with responsibility for key ventilation and weaning decisions and to examine organizational characteristics associated with nurse involvement.
\end{abstract}

Methods: A multi-center, cross-sectional, self-administered survey was sent to nurse managers of adult intensive care units (ICUs) in Denmark, Germany, Greece, Italy, Norway, Switzerland, Netherlands and United Kingdom (UK). We summarized data as proportions ( $95 \%$ confidence intervals $(\mathrm{Cls})$ ) and calculated odds ratios (OR) to examine ICU organizational variables associated with collaborative decision making.

Results: Response rates ranged from 39\% (UK) to 92\% (Switzerland), providing surveys from 586 ICUs. Interprofessional collaboration (nurses and physicians) was the most common approach to initial selection of ventilator settings (63\% (95\% Cl 59 to 66)), determination of extubation readiness (71\% (67 to 75$)$ ), weaning method (73\% (69 to 76)), recognition of weaning failure (84\% (81 to 87$))$ and weaning readiness (85\% (82 to 87$)$ ), and titration of ventilator settings (88\% (86 to 91)). A nurse-to-patient ratio other than 1:1 was associated with decreased interprofessional collaboration during titration of ventilator settings (OR $0.2,95 \% \mathrm{Cl} 0.1$ to 0.6$)$, weaning method (0.4 (0.2 to 0.9)), determination of extubation readiness (0.5 (0.2 to 0.9)) and weaning failure (0.4 (0.1 to 1.0)). Use of a weaning protocol was associated with increased collaborative decision making for determining weaning (1.8 (1.0 to 3.3)) and extubation readiness (1.9 (1.2 to 3.0)), and weaning method (1.8 (1.1 to 3.0). Country of ICU location influenced the profile of responsibility for all decisions. Automated weaning modes were used in $55 \%$ of ICUs.

Conclusions: Collaborative decision making for ventilation and weaning was employed in most ICUs in all countries although this was influenced by nurse-to-patient ratio, presence of a protocol, and varied across countries. Potential clinical implications of a lack of collaboration include delayed adaptation of ventilation to changing physiological parameters, and delayed recognition of weaning and extubation readiness resulting in unnecessary prolongation of ventilation.

\footnotetext{
* Correspondence: louise.rose@utoronto.ca

'Lawrence S. Bloomberg Faculty of Nursing, University of Toronto, 155

College St, Toronto, M5T 1P8, Canada

Full list of author information is available at the end of the article
}

\section{Biomed Central}




\section{Introduction}

Optimal management of mechanical ventilation and weaning requires dynamic and collaborative decision making to minimize complications and avoid delays in the transition to extubation. Effective collaboration requires open, extensive, and coordinated communication as well as shared team goals and will result in improved quality of care, patient safety and discharge outcomes [1-3]. In the absence of collaboration, ventilation decision making may be fragmented, inconsistent, and delayed [4].

Previous studies exploring interprofessional responsibility for ventilation and weaning decision making in Australia, New Zealand, and Denmark found that physicians and nurses actively collaborated in the management of ventilation and weaning, generally in the absence of protocols [5-8]. Nurses in Australia and New Zealand were frequently independently responsible for manipulation of ventilator settings titrated to physiologic parameters [9]. Intensive care unit (ICU) organizational characteristics have been noted as key contributors to ICU performance and patient outcomes [10]. Organizational characteristics such as staffing ratios, hierarchical structure, and ICU team functioning in the above countries may differ from those elsewhere [11].

International variation in aspects of the delivery of mechanical ventilation such as preferred ventilator mode, use of non-invasive ventilation (NIV), and adoption of protocols for weaning has been noted previously $[12,13]$. Interprofessional roles and responsibilities are influenced by differences in unit structure, staffing and skill-mix, patient case-mix, and medical and nursing leadership models [14]. The primary objective of our study was to describe the professional group with responsibility for determining key ventilation and weaning decisions including: selection of initial ventilator settings, titration of ventilator settings, weaning readiness, weaning method, extubation readiness, and weaning failure. We hypothesized that substantial variation would exist between and within countries for the professional group responsible for these decisions.

Our secondary objectives were to examine organizational characteristics associated with nurse involvement in ventilator and weaning decision making including nurse-to-patient ratios for invasive and non-invasive ventilation; perceived nursing autonomy and nursing contribution to ventilator decision-making; to describe the use of automated systems, protocols and/or guidelines for the management of mechanical ventilation and weaning; and the availability of education related to mechanical ventilation and associated processes for nurses. Results from the Netherlands have been previously reported in abstract form [15].

\section{Materials and methods Study Design and Sampling Frame}

We conducted a multi-center, cross sectional, self-administered survey of adult ICUs in Denmark, Germany, Greece, Italy, Norway, Switzerland, the Netherlands and the United Kingdom (UK). These countries represent three of the four European sub-regions: Northern, Western, and Southern Europe. Our sample frame comprised all ICUs providing mechanical ventilation to critically ill adults in Denmark, Norway, Switzerland, the UK, and the Netherlands identified through existing intensive care networks. For example, ICUs in the UK were identified using the 2008 Directory of Critical Care [16]. Telephone contact was made to confirm that the ICU met inclusion criteria and to obtain nurse manager contact details. In Germany, Greece, and Italy we were unable to identify a reliable comprehensive list of all adult ICUs. Therefore nurse managers were recruited from existing personal e-mail lists within regions of a country (Attica in Greece and the Piedmont and Valle D'Aosta Regions in Italy) and nationally via advertisements in local journals and websites.

\section{Study Population}

We included nurse managers of adult ICUs providing care to ventilated patients. We requested nurse managers consult senior medical colleagues and other senior nurses involved in the clinical management of ventilated patients to provide the best possible reflection of ventilation and weaning processes within their unit. If more than one nurse manager was employed in an ICU, we requested that only one survey be returned that reflected a consensus of opinion. Pediatric and neonatal ICUs or units not routinely providing mechanical ventilation such as coronary care and high dependency units were excluded.

\section{Survey Development and Testing}

We administered a survey of mechanical ventilation and weaning responsibilities previously described and conducted in Australia and New Zealand [5]. Due to increased commercial availability of automated closed loop systems $[17,18]$, we added a question on the use of SmartCare/PS ${ }^{\mathrm{TM}}$ (Dräger Medical, Lübeck, Germany), Adaptive Support Ventilation (ASV) (Hamilton Medical, Bonaduz, Switzerland), Proportional Assist Ventilation (PAV), and Mandatory Minute Ventilation (MMV). Questions relating to ICU demographic and staffing descriptions were contextually adapted for each country based on input from senior nurses and physicians. The survey (see Additional file 1) was then forward and back translated into the required languages. Country coordinators (fluent in English and the native language) and 
the principal investigator resolved inconsistencies in the two English versions (initial version and back-translated). Once the two English versions were consistent, the translated version was revised and checked by another native speaker. Prior to distribution, face validity was assessed in each country by a panel of experienced ICU nurses and physicians.

\section{Data Collection}

Approval for conduct of the survey was obtained from ethics review boards and/or hospital administration in each country as required according to local guidelines. Return of a completed questionnaire was considered indicative of consent. Participants were advised that survey completion was voluntary. To maintain anonymity, no ICU or participant identifiers were collected.

A study investigator coordinated survey administration in each country. Surveys were distributed via mail in the UK and the Netherlands; by email in Denmark, Switzerland, Germany, Italy and Norway; and conducted by phone in Greece. In Germany a link to the survey was also advertised on professional websites. Country coordinators selected the survey delivery method based on available contact details. Three reminders to complete the survey were sent via mail, email or phone at two week intervals from initial distribution. Participants who received mail surveys were provided with a stampedaddressed envelope to return the survey to the coordinating center of that country. Email surveys were returned to the country coordinator (Denmark, Switzerland and Italy) or to a secure collector maintained by either Global Park http://www.globalpark.com/ (Germany) or Questback http://www.questback.com/ (Norway).

\section{Statistical Methods}

We included data from incomplete questionnaires, therefore denominators for survey items vary. We excluded from analysis surveys with $>50 \%$ incomplete items. We summarized categorical variables such as professional group responsible for ventilation decisions using proportions and their 95\% confidence intervals (CI). We calculated relative risks to determine the ventilator settings most likely to be adjusted by nurses $>50 \%$ of the time. The total scores for each of the two 0 to 10 scales used to measure perceived autonomy and nursing contribution to decision-making ( 0 represented no nurse autonomy and decision making input and 10 represented complete autonomy and nurse input into all decisions) were calculated and the median and interquartile range (IQR) determined.

Relevant variables selected $a$ priori (country, nurse-topatient ratio, presence of a protocol, hospital teaching status, number of ICU beds, and open versus closed model ICU) as likely to be associated with the professional group (collaborative compared to medical input only) most responsible for each of the six key decisions were examined using multiple logistic regression and odds ratios and their 95\% CIs calculated. All models were assessed for collinearity and goodness of fit. All tests were two-tailed and we considered a $P$-value of 0.05 as statistically significant. Analyses were performed using SPSS 18.0 (SPSS, Chicago, IL, USA).

\section{Results}

\section{Response Rates and Unit Characteristics}

Response rates ranged from 39\% (UK) to 92\% (Switzerland) providing 586 surveys for evaluation. We were unable to determine the participant denominator in Germany due to the recruitment methods used and therefore cannot report a response rate. Unit characteristics are shown in Table 1. Germany had a higher proportion of ICUs with $>8$ beds and more open ICUs than other countries except Denmark. A 1:1 nurse-topatient ratio was used almost exclusively ( $93 \%$ of ICUs) for invasively ventilated patients in the UK; $6 \%$ used either a 1:1 or 1:2 nurse-to-patient ratio dependent on patient acuity. Switzerland, Denmark and Norway employed a 1:1 nurse-to-patient ratio in the majority of ICUs $(61 \%, 73 \%$ and $90 \%$, respectively). In the remaining countries, a 1:2 nurse-to-patient ratio was most common. For patients receiving NIV, a 1:2 nurse-to-patient ratio was most common in Germany, Switzerland, Italy, the Netherlands and the UK. ICUs in Denmark and Norway used a 1:1 nurse-to-patient ratio and Greek ICUs a 1:3 ratio.

\section{Decisional Responsibility}

Interprofessional responsibility for six key ventilation and weaning decisions is shown by country in Table 2 . Interprofessional collaboration was the most common approach for all decisions regarding ( $\mathrm{n} / \mathrm{N}, \%(95 \% \mathrm{CI})$ ) initial selection of ventilator settings (365/584, 63\% (5966)), determination of extubation readiness $(414 / 581$, $71 \%$ (67-75)), weaning method (423/583, 73\% (69-76)), recognition of weaning failure $(489 / 582,84 \%(81-87))$ and weaning readiness $(496 / 585,85 \%(82-87))$, and titration of ventilator settings (515/582, 88\% (86-91)). Despite interprofessional collaboration being least likely in the selection of initial ventilator settings, nurses collaborated in this decision in $>75 \%$ of respondent ICUs in Switzerland, Germany and the UK.

A nurse-to-patient ratio other than 1:1 was associated with decreased interprofessional collaboration (decisions made independently by physicians without nursing input) during titration of ventilator settings (OR 0.2 (95\% CI 0.1$0.6)$ ), weaning method $(0.4(0.2-0.9))$, determination of extubation readiness $(0.5(0.2-0.9))$ and weaning failure 
Table 1 ICU Demographics

\begin{tabular}{|c|c|c|c|c|c|c|c|c|}
\hline & $\begin{array}{l}\text { Switzerland } \\
(n=73)\end{array}$ & $\begin{array}{c}\text { UK } \\
(n=115)\end{array}$ & $\begin{array}{l}\text { Germany } \\
(n=201)\end{array}$ & $\begin{array}{l}\text { Netherlands } \\
(n=71)\end{array}$ & $\begin{array}{l}\text { Denmark } \\
(n=41)\end{array}$ & $\begin{array}{c}\text { Greece } \\
(n=12)\end{array}$ & $\begin{array}{l}\text { Norway } \\
(n=39)\end{array}$ & $\begin{array}{c}\text { Italy } \\
(n=34)\end{array}$ \\
\hline \multicolumn{9}{|l|}{ Hospital type } \\
\hline Private & $6(6)$ & - & & - & - & - & - & - \\
\hline University affiliated & $11(15)$ & $71(62)$ & $64(32)$ & $3(4)$ & $22(54)$ & $5(42)$ & $11(28)$ & $1(3)$ \\
\hline Community/cantonal (teaching) & $47(64)$ & $18(16)$ & $135(67)$ & $43(61)$ & $9(22)$ & $3(25)$ & $28(72)$ & $23(68)$ \\
\hline $\begin{array}{l}\text { Community/cantonal } \\
\text { (non-teaching) }\end{array}$ & $9(12)$ & $21(18)$ & - & $22(31)$ & $10(24)$ & $4(33)$ & - & $10(29)$ \\
\hline \multicolumn{9}{|l|}{ ICU specialty } \\
\hline Med/surg/trauma & $34(47)$ & $59(51)$ & $49(24)$ & $29(41)$ & $15(37)$ & $5(42)$ & $18(46)$ & $21(62)$ \\
\hline Medical/surgical & $26(36)$ & $36(31)$ & $50(25)$ & $33(47)$ & $16(39)$ & $3(25)$ & $12(31)$ & $4(12)$ \\
\hline Medical (only) & $5(7)$ & - & $38(19)$ & - & $2(5)$ & - & $2(5)$ & $2(6)$ \\
\hline Surgical (only) & $3(4)$ & $3(3)$ & $24(12)$ & $2(3)$ & - & - & $3(8)$ & $2(6)$ \\
\hline Cardiothoracic & $1(1)$ & $8(7)$ & $14(7)$ & $1(1)$ & $3(7)$ & $4(33)$ & $1(3)$ & $4(12)$ \\
\hline Neuro/trauma & $3(4)$ & $4(4)$ & $15(8)$ & $2(3)$ & $2(5)$ & - & $1(3)$ & - \\
\hline Neurosurgery (only) & $1(1)$ & - & $5(3)$ & - & $3(7)$ & - & $1(3)$ & - \\
\hline Burns & - & $1(1)$ & $1(1)$ & - & - & - & $1(3)$ & - \\
\hline \multicolumn{9}{|l|}{ ICU type } \\
\hline closed & $66(90)$ & $97(84)$ & $142(71)$ & $44(62)$ & $9(22)$ & $7(58)$ & $30(77)$ & $32(94)$ \\
\hline open & $6(8)$ & $12(10)$ & $56(28)$ & $15(21)$ & $22(54)$ & $3(25)$ & $8(21)$ & $1(3)$ \\
\hline \multicolumn{9}{|l|}{ ICU bed numbers } \\
\hline$\leq 8$ beds & $44(60)$ & $59(51)$ & $36(18)$ & $34(48)$ & $19(46)$ & $5(42)$ & $25(64)$ & $28(82)$ \\
\hline $9-16$ beds & $18(25)$ & $31(27)$ & $116(58)$ & $26(37)$ & $11(27)$ & $6(50)$ & $7(18)$ & $6(18)$ \\
\hline$\geq 17$ beds & $7(10)$ & $16(21)$ & $48(24)$ & $9(13)$ & $8(20)$ & $1(8)$ & $5(13)$ & \\
\hline
\end{tabular}

All data are $\mathrm{n}$ (\%). Percentages may not add up to $100 \%$ due to missing data or rounding. Med, medical; $\mathrm{n}$, number; surg, surgical.

(0.4 (0.1-1.0)) when controlling for country, ICU type (open versus closed), ICU size, presence of a protocol, and hospital teaching status. Use of a ventilator protocol or guideline was associated with increased collaborative decision making (communication between physicians and nurses) for weaning (OR 1.8 (95\% CI 1.0-3.3)) and extubation readiness $(1.9(1.2-3.0))$ and weaning method (1.8 (1.1-3.0)) when controlling for the same variables. Country of ICU location influenced the profile of professional responsibility for all decisions. Nurses were least likely to be involved in any type of ventilator decision making in ICUs located in Greece and Italy and most likely to be involved in Switzerland and the UK. Nurses were independently (without consulting a physician) responsible for titration of Fraction of inspired oxygen $\left(\mathrm{FiO}_{2}\right)$ and pressure support in most ICUs (Table 3 ). In the majority of ICUs in Switzerland, Germany and the UK, nurses independently titrated all ventilator settings including change of mode except the level of positive end expiratory pressure (PEEP). Nurses rarely independently titrated ventilator settings in Italy and Greece.

\section{Automated Closed Loop Systems and Ventilation Protocols}

Of the 586 ICUs, 319 (55\% [50-59]) indicated they used SmartCare/PS, ASV, PAV or MMV. More ICUs reported using ASV > 50\% of the time than other automated weaning systems (Figure 1). Protocols for ventilation (54\% in the UK to $81 \%$ in Switzerland) and weaning (56\% in Italy to $69 \%$ in Switzerland) were used in most ICUs in all countries with the exception of Greece where no ICU reported a protocol for management of ventilation and only one ICU reported availability of a weaning protocol. Availability of protocols for NIV ranged from 1 in 12, 8\% (Greece) to 62 in 71, 87\% (Netherlands) ICUs.

\section{Nurse Autonomy, Influence and Ventilator Education}

Nurse managers' ratings of nurse autonomy and influence on decision making about mechanical ventilation practices ranged from 0 (no autonomy or influence) to 10 (complete autonomy and always influenced decisions) with a median score of 7 for both scales. Autonomy was rated highest by Swiss nurse managers (median 8, IQR 6 to 8) and lowest by those from Greek ICUs (5, 5 to 7). Ratings of nurse influence in ventilation decision making were similar across all countries. Most ICUs in all countries provided education related to ventilation to nurses during commencement of employment (65\% in Italy [lowest] to $98 \%$ in the UK [highest]).

\section{Discussion}

Our findings indicate, according to nurse managers, that interprofessional collaboration was the predominant 
Table 2 Responsibility for Ventilation Decisions by Country

\begin{tabular}{|c|c|c|c|c|c|c|c|c|}
\hline & $\begin{array}{c}\text { Switzerland } \\
(n=73)\end{array}$ & $\begin{array}{c}\text { UK } \\
(n=115)\end{array}$ & $\begin{array}{l}\text { Germany } \\
(n=201)\end{array}$ & $\begin{array}{l}\text { Netherlands } \\
(n=71)\end{array}$ & $\begin{array}{l}\text { Denmark } \\
(n=41)\end{array}$ & $\begin{array}{c}\text { Greece } \\
(n=12)\end{array}$ & $\begin{array}{l}\text { Norway } \\
(n=39)\end{array}$ & $\begin{array}{c}\text { Italy } \\
(n=34)\end{array}$ \\
\hline \multicolumn{9}{|c|}{ Initial ventilator settings } \\
\hline Physician only & $13(18)$ & $23(20)$ & $49(24)$ & $32(45)$ & $18(44)$ & $7(58)$ & $25(64)$ & $23(68)$ \\
\hline Collaborative & $55(85)$ & $90(78)$ & $134(67)$ & $40(56)$ & $22(54)$ & $5(42)$ & $14(36)$ & $11(32)$ \\
\hline Nurse only & $4(6)$ & $1(1)$ & $18(9)$ & - & - & - & - & - \\
\hline Other & $1(1)$ & - & - & $1(1)$ & - & - & - & - \\
\hline \multicolumn{9}{|c|}{ Titration of ventilator settings } \\
\hline Physician only & $3(4)$ & - & $8(4)$ & $3(4)$ & $2(5)$ & $1(8)$ & $3(8)$ & $15(44)$ \\
\hline Collaborative & $63(86)$ & $112(99)$ & $176(88)$ & $66(93)$ & $37(90)$ & $11(92)$ & $34(87)$ & $19(56)$ \\
\hline Nurse only & $5(6.8)$ & $1(1)$ & $15(8)$ & $2(3)$ & $1(2)$ & - & $2(5)$ & - \\
\hline Other & $2(3)$ & $1(1)$ & - & - & - & - & - & - \\
\hline \multicolumn{9}{|l|}{ Readiness to wean } \\
\hline Physician only & $5(7)$ & $4(4)$ & $25(12)$ & $11(16)$ & $1(2)$ & $8(67)$ & $7(18)$ & $18(53)$ \\
\hline Collaborative & $65(89)$ & $111(97)$ & $172(82)$ & $59(83)$ & $39(95)$ & $4(33)$ & $32(82)$ & $16(47)$ \\
\hline Nurse only & $2(3)$ & - & $3(2)$ & - & - & - & - & - \\
\hline Other & $1(1)$ & $4(4)$ & - & $1(1)$ & - & - & - & - \\
\hline \multicolumn{9}{|l|}{ Weaning method } \\
\hline Physician only & $5(7)$ & $10(9)$ & $37(18)$ & $20(28)$ & $10(24)$ & $6(50)$ & $11(28)$ & $25(74)$ \\
\hline Collaborative & $59(81)$ & $104(90)$ & $131(71)$ & $49(69)$ & $31(76)$ & $6(50)$ & $27(69)$ & $9(27)$ \\
\hline Nurse only & $4(6)$ & - & $22(11)$ & $1(1)$ & - & - & - & - \\
\hline Other & $5(7)$ & $6(5)$ & - & $1(1)$ & - & - & - & - \\
\hline \multicolumn{9}{|c|}{ Readiness to extubate } \\
\hline Physician only & $17(23)$ & $15(13)$ & $45(22)$ & $35(49)$ & $9(22)$ & $7(58)$ & $13(33)$ & $20(59)$ \\
\hline Collaborative & $56(77)$ & $98(85)$ & $152(76)$ & $36(49)$ & $32(78)$ & $5(42)$ & $25(64)$ & $14(41)$ \\
\hline Nurse only & - & - & $2(1)$ & - & - & - & - & - \\
\hline Other & - & $2(2)$ & - & - & - & - & - & - \\
\hline \multicolumn{9}{|l|}{ Weaning failure } \\
\hline Physician only & $7(10)$ & $2(2)$ & $13(7)$ & $7(10)$ & $4(10)$ & $6(50)$ & $6(15)$ & $12(35)$ \\
\hline Collaborative & $65(89)$ & $105(91)$ & $169(85)$ & $57(80)$ & $37(90)$ & $6(50)$ & $32(82)$ & $21(62)$ \\
\hline Nurse only & $1(1)$ & $5(4)$ & $17(9)$ & $3(4)$ & - & - & - & - \\
\hline Other & - & $2(2)$ & - & $4(6)$ & - & - & - & - \\
\hline
\end{tabular}

All data are $\mathrm{n}(\%)$. Note some $\%$ do not add to 100 due to missing data. Other: includes physiotherapists or specialized ventilator practitioners who may also hold a nursing qualification. $n$, number

Table 3 Type of Ventilator Settings made independently by Nurses*

\begin{tabular}{lccc}
\hline & $\mathbf{n} / \mathbf{N}$ & \% (95\% Cl) & RR (95\% Cl) \\
\hline Decrease of $\mathrm{FiO}_{2}$ & $392 / 580$ & $68(64-71)$ & 1 \\
Increase of $\mathrm{FiO}_{2}$ & $386 / 580$ & $67(63-70)$ & $1.0(0.9-1.1)$ \\
Increase of pressure support & $321 / 582$ & $55(51-59)$ & $0.8(0.7-0.9)$ \\
Decrease of pressure support & $317 / 580$ & $55(51-59)$ & $0.8(0.7-0.9)$ \\
Titration of respiratory rate & $290 / 581$ & $50(46-54)$ & $0.7(0.7-0.8)$ \\
Titration of tidal volume & $251 / 576$ & $44(40-48)$ & $0.6(0.6-0.7)$ \\
Titration of inspiratory pressure & $229 / 577$ & $40(36-44)$ & $0.6(0.5-0.7)$ \\
Change mode & $231 / 583$ & $40(36-44)$ & $0.6(0.5-0.7)$ \\
Decrease of PEEP & $162 / 579$ & $28(25-32)$ & $0.4(0.4-0.5)$ \\
Increase of PEEP & $147 / 581$ & $25(22-29)$ & $0.4(0.3-0.4)$ \\
\hline
\end{tabular}

*Nurse responsible to make and implement ventilator setting change independent $>50 \%$ of the time.

$\mathrm{Cl}$, confidence interval; $\mathrm{RR}$, relative risk; $\mathrm{FiO}_{2}$, fraction of inspired oxygen; number; PEEP, positive end expiratory pressure. model for decisions about mechanical ventilation and weaning and nurses generally had a reasonable influence on decisions made. Interprofessional collaboration varied according to the type of decision with physicians more likely to select initial ventilator settings and nurses more involved in the ongoing titration of ventilation and determination of extubation readiness. To the best of our knowledge, this is the largest survey describing interprofessional role responsibility for mechanical ventilation across Europe. Our findings suggest greater involvement of nurses in ventilator adjustment compared to a previous survey of physicians profiling ICU nursing in Western Europe conducted over a decade ago [14] but congruence in some countries with interprofessional responsibility for ventilation decisions in Australia and New Zealand [5] and participation in weaning practices reported in a pilot study of European nurses [19]. Physicians are generally present during 


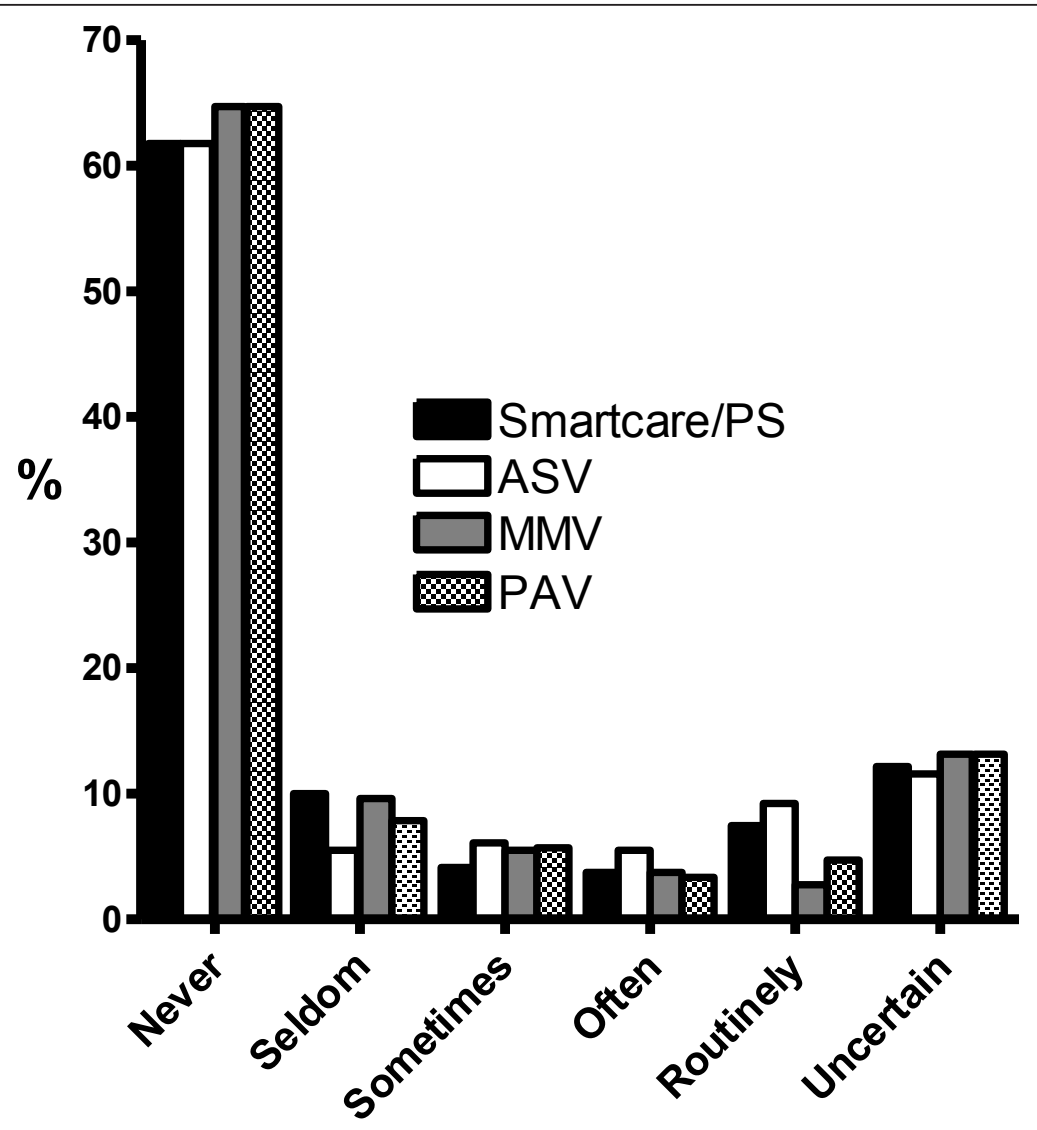

Figure 1 Use of Automated Closed Loop Systems.

initiation of ventilation as it coincides with intubation or arrival in ICU and represents an acute deterioration in respiratory status. ICU nurses maintain a near continuous presence at the bedside and therefore may be best positioned to titrate ventilator settings in response to changes in physiologic parameters.

Interprofessional collaboration for ventilation decision making varied by country with nursing input into ventilation decisions highest in Switzerland, Germany, and the UK and lowest in Greece and Italy. Irrespective of country, collaboration was influenced by nurse-to-patient ratio and the presence of protocols. Variation in the degree of collaboration is likely due to organizational, professional, and systemic factors that create power differences and delineate role responsibility [20]. The clinical implications of this variation in collaborative decision making on patient outcomes such as the duration of ventilation and weaning is unclear. However, several randomized controlled trials of either weaning or sedation protocols attribute the lack of reduction in ventilation duration to the existing organizational context including high staffing ratios, nurse autonomy in decision making and frequency of medical rounds that influence the usual care arm of the trial [21-23]. The absence of interprofessional collaboration has the potential to result in delayed adaptation of ventilation to changing physiological parameters, and delayed recognition of weaning and extubation readiness resulting in unnecessary prolongation of ventilation. High levels of interprofessional collaboration have previously been associated with low standardized mortality ratios (SMR) [3] and lower rates of ICU readmission following ICU discharge [24,25]; whereas ineffective interprofessional collaboration has been associated with the development of ventilator associated pneumonia and pressure ulcers [26], poor team functioning, morale [27] and ethical decision making [28]. Further studies are required to examine the impact of interprofessional collaboration on potentially modifiable outcomes such as weaning duration.

The utility of nurse involvement in ventilator decision making is reliant on appropriate knowledge and skills to manage ventilation. Differences existed in the proportion of ICUs providing education related to mechanical ventilation across countries, most probably as a reflection on the role of nurses in ventilation and weaning. On a broader level, differences also exist in the type and content of critical care nursing education internationally [29] that likely impact nurse involvement and autonomy. Furthermore, in ICUs with lower nurse-to-patient ratios, 
nurses may not be available for ventilator decision making due to the demands of ongoing assessment and provision of care to more than one patient.

Our findings suggest that nurses were more likely to make and implement decisions related to weaning, such as titration of pressure support and $\mathrm{FiO}_{2}$, independently. Nursing involvement in weaning has increased over the past two decades with the introduction of weaning protocols. Although, as discussed above, the effect of weaning protocols may be moderated by contextual factors [30] and no study has identified harm associated with nursing involvement in the weaning process. Interestingly, the level of PEEP was the ventilator setting least likely to be independently adjusted by nurses. While we did not seek information on the rationales for nurse initiated ventilator titration, we hypothesize that knowledge required to adjust PEEP may be viewed as more complex or that PEEP adjustment is perceived to pose greater risk of adverse consequences, although the logic of this viewpoint is questionable given the evidence of harm related to high tidal volume ventilation [31].

This study, to the best of our knowledge, is the first to report insight into the use of automated weaning systems in practice. Though just over $50 \%$ of ICUs reported using one or more automated closed loop system, few ICUs in all countries used them routinely. The goal of automated weaning systems is to improve adaptation of ventilatory support to the patients' needs through continuous monitoring and real-time interventions [17]. Several randomized controlled trials describe a reduction in duration of mechanical ventilation using automated weaning [32-34] while others demonstrate no effect $[23,35]$. As technology advances and the evidence about the utility of these systems increases, it is important to continue to track adoption into practice.

Our study has several limitations including the potential for selection bias, self-report bias, confounding, and lack of generalizability. Nurse managers interested in the delineation of interprofessional responsibilities for mechanical ventilation were probably more likely to respond to this questionnaire. As we chose to survey nurse managers, nurses' roles with respect to ventilation and weaning may be over-represented despite our instruction to confer with senior medical and nursing colleagues. There is also the possibility that other contextual factors not measured in our survey contributed to decisional responsibility. Lack of generalizability is particularly problematic regarding survey responses from Greece and Italy as only certain regions of these countries were surveyed.

\section{Conclusion}

Our cross-sectional survey of nurse managers suggests that collaborative decision making for important decisions related to ventilation and weaning was employed in most ICUs in all countries. Nurse-topatient ratio, availability of protocols and country influenced nurse involvement in decision making. Further study is warranted to determine if collaborative decision making is associated with improved patient outcomes for mechanically ventilated patients.

\section{Key messages}

- interprofessional collaboration was the predominant model for decisions about mechanical ventilation and weaning

- interprofessional collaboration for ventilation decision making varied by country and was modified by nurse-to-patient ratio and the presence of protocols - more than $50 \%$ of ICUs reported using one or more automated closed loop system, few ICUs in all countries used them routinely

- variation exists in the adoption of protocols for ventilation and weaning

\section{Additional material}

Additional file 1: Survey of Ventilation and Weaning Responsibility. Generic version of the survey in English.

\section{Abbreviations}

ASV: adaptive support ventilation; Cl: confidence interval; ICU: intensive care unit; IQ: interquartile range; MMV: mandatory minute ventilation; NIV non invasive ventilation; OR: odds ratio; PAV: proportional assist ventilation; PEEP: positive end expiratory pressure.

\section{Acknowledgements}

The authors thank Catherine Kelly, Mette Juel Blichfeldt, Anouk Kanitz, and Aly Hovingh for their assistance in the completion of this study.

\section{Author details}

${ }^{1}$ Lawrence S. Bloomberg Faculty of Nursing, University of Toronto, 155 College St, Toronto, M5T 1P8, Canada. ${ }^{2}$ Department of Respirology, Toronto East General Hospital, 825 Coxwell Ave, Toronto, M4C 3E7, Canada. ${ }^{3}$ Department of Nursing, Mt Sinai Hospital, 600 University Ave, Toronto, M5G 1X5, Canada. ${ }^{4}$ Li Ka Shing Institute, St Michael's Hospital, 30 Bond St, Toronto, M5B 1W8, Canada. ${ }^{5}$ School of Nursing and Midwifery, Queen's University, 97 Lisburn Road, Belfast, BT9 7BL, Northern Ireland. ${ }^{6}$ The University of Copenhagen, Faculty of Health Sciences and The University Hospitals Center for Nursing and Care Research, UCS, Copenhagen University Hospital Rigshospitalet, Department 7001, Blegdamsvej 9, DK-2100 Copenhagen O, Denmark. ${ }^{7}$ Department for Research and Development, Levanger Hospital, Nord-Trøndelag Health Trust, Kirkegt. 2, Levanger, 7600, Norway. ${ }^{8}$ Department of Intensive Care, Gelre Hospitals, Albert

Schweitzerlaan 31, Apeldoorn, 7334DZ, The Netherlands. ${ }^{9}$ Deutsches Institut für angewandte Pflegeforschung, Hülchrather Str. 15 Köln, 50670, Germany. ${ }^{10}$ School of Health in Social Science Nursing Studies, The University of Edinburgh, 17/10 High Riggs, Edinburgh, EH3 9BW UK. ${ }^{11}$ Faculty of Medicine, University of Basel, Institute of Nursing Science, Bernoullistr. 28, Basel 4056, Switzerland. ${ }^{12}$ Center of Clinical Nursing Science, University Hospital Zurich, Raemistr. 100 (ZUR 44), Zurich 8091, Switzerland. ${ }^{13}$ Faculty of Medicine and Surgery, Catholic University of the Sacred Heart, School of Nursing, Largo Francesco Vito, 1- 00168 Roma, Italy. ${ }^{14}$ Cottolengo Hospital, Little House of Divine Providence, S.C. Training and Quality, School of Nursing, via Cottolengo 13, Turin, 10100, Italy. ${ }^{15}$ Department of Intensive Care, Gelre 
Hospitals, Albert Schweitzerlaan 31, Apeldoorn, 7334DZ, The Netherlands. ${ }^{16}$ Department of Health and Care Sciences, Faculty of Health Sciences, University of Tromsø, Romssa universitehta, N-9037, Norway. ${ }^{17}$ Centre for Infection and Immunity, Room 01/017, Health Sciences Building, Queen's University, 97 Lisburn Road, Belfast BT9 7BL, Northern Ireland. ${ }^{18}$ Department of Intensive Care Medicine, Academic Medical Center at the University of Amsterdam, Meibergdreef 9, Amsterdam, 1105 AZ, The Netherlands.

\section{Authors' contributions}

LR, MJS, PS, IE, BB, HH conceived the study. BB, DM, IE, HH, JH, MI, KK, MS, RS coordinated data collection and data entry in their own countries. LR collated and analyzed the data. All authors contributed to manuscript drafts and have read and approved the final manuscript.

\section{Competing interests}

The authors declare that they have no competing interests.

Received: 23 August 2011 Revised: 14 October 2011

Accepted: 14 December 2011 Published: 14 December 2011

\section{References}

1. Reader T, Flin R, Cuthbertson B: Team leadership in the intensive care unit: the perspective of specialists. Crit Care Med 2011, 39:1683-1691.

2. Reader $T$, Flin R, Mearns $K$, Cuthbertson B: Interdisciplinary communication in the intensive care unit. Br J Anaesth 2007, 98:347-352.

3. Wheelan S, Burchill C, Tilin F: The link between teamwork and patients' outcomes in intensive care units. Am J Crit Care 2003, 12:527-534.

4. Hansen B, Severinsson E: Physicians' perceptions of protocol-directed weaning in an intensive care unit in Norway. Nurs Health Sci 2009 11:71-76.

5. Rose L, Nelson S, Johnston L, Presneill J: Workforce profile, organisation structure and role responsibility for ventilation and weaning practices in Australia and New Zealand intensive care units. J Clin Nur 2008, 17:1035-1043.

6. Egerod I: Mechanical ventilator weaning in the context of critical care nursing A descriptive, comparative study of nurses' decisions and interventions related to mechanical ventilator weaning Copenhagen: University of Copenhagen; 2003.

7. Hansen B, Fjaelberg W, Nilsen O, Lossius H, Soreide E: Mechanical ventilation in the ICU-is there a gap between the time available and time used for nurse-led weaning? Scand J Trauma Resusc Emerg Med 2008, 16:17.

8. Egerod I, Christensen B, Johansen L: Trends in sedation practices in Danish intensive care units in 2003: a national survey. Intensive Care Med 2006, 32:60-66.

9. Rose L, Nelson $S$, Johnston L, Presneill J: Decisions made by critical care nurses during mechanical ventilation and weaning in an Australian intensive care unit. Am J Crit Care 2007, 16:434-443.

10. Nguyen $Y$, Wunsch $H$, Angus $D$ : Critical care: the impact of organization and management on outcomes. Curr Opin Crit Care 2010, 16:487-492.

11. Bellomo R, Stow P, Hart G: Why is there such a difference in outcome between Australian intensive care units and others? Curr Opin Anaesthesiol 2007, 20:100-105.

12. Esteban A, Ferguson ND, Meade MO, Frutos-Vivar F, Apezteguia C, Brochard L, Raymondos K, Nin N, Hurtado J, Tomicic V, González M, Elizalde J, Nightingale P, Abroug F, Pelosi P, Arabi Y, Moreno R, Jibaja M, D'Empaire G, Sandi F, Matamis D, Montañez AM, Anzueto A, VENTILA Group: Evolution of mechanical ventilation in response to clinical research. Am J Respir Crit Care Med 2008, 177:170-177.

13. Metnitz P, Metnitz B, Moreno R, Bauer P, Del Sorbo L, Hoermann C, de Carvalho S, Ranieri V, SAPS 3 Investigators: Epidemiology of mechanical ventilation: analysis of the SAPS 3 database. Intensive Care Med 2009, 35:816-825.

14. Depasse B, Pauwels D, Somers $Y$, Vincent J: A profile of European ICU nursing. Intensive Care Med 1998, 24:939-945.

15. Kanitz A, Hofhuis J, Schultz M, Rose L, Spronk P: Role-responsibilities for mechanical ventilation and weaning in the Netherlands. Intensive Care Med 2009, 35:S203.

16. CMA Medical Data: Directory of Critical Care 2008. Loughborough UK: CMA Medical Data; 2008
17. Lellouche F, Brochard L: Advanced closed loops during mechanical ventilation (PAV, NAVA, ASV, SmartCare). Best Pract Res Clin Anaesthesiol 2009, 23:81-93.

18. Rose L, Presneill J, Cade J: Update in computer-driven weaning from mechanical ventilation. Anaesth Intensive Care 2007, 35:213-221.

19. Benbenishty J, DeKeyser Ganz F, Adam S: Differences in European critical care nursing practice: a pilot study. Intensive Crit Care Nurs 2005, 21:172-178.

20. San Martin Rodriguez L, Beaulieu M-D, D'Amour D, Ferrada-Videla M: The determinants of successful collaboration: a review of theoretical and empirical studies. J Interprof Care 2005, 19:132-147.

21. Krishnan JA, Moore D, Robeson C, Rand CS, Fessler HE: A prospective, controlled trial of a protocol-based strategy to discontinue mechanical ventilation. Am J Respir Crit Care Med 2004, 169:673-678.

22. Bucknall T, Manias E, Presneill J: A randomized trial of protocol-directed sedation management for mechanical ventilation in an Australian intensive care unit. Crit Care Med 2008, 36:1444-1450.

23. Rose $L$, Presneill J, Johnston $L$, Cade J: A randomised, controlled trial of conventional weaning versus an automated system (SmartCare ${ }^{\mathrm{TM}} / \mathrm{PS}$ in mechanically ventilated critically-ill patients. Intensive Care Med 2008, 34:1788-1795.

24. Baggs J, Ryan S, Phelps C, Richeson J, Johnson J: The association between interdisciplinary collaboration and patient outcomes in a medical intensive care unit. Heart Lung 1992, 21:18-24.

25. Baggs J, Schmitt M, Mushlin A, Mitchell P, Eldrege D, Oakes D: Association bewteen nurse-physician collaboration and patient outcomes in three intensive care units. Crit Care Med 1999, 27:1991-1998.

26. Manojlovich M, Anatonakos C, Ronis D: Intensive care units, communication between nurses and physicians, and patient outcomes. Am J Crit Care 2009, 18:21-30

27. Baggs J, Schmitt M, Mushlin A, Eldredge D, Oakes D, Hutson A: Nurses and resident physicians' perceptions of the process of collaboration in an MICU. Res Nurs Health 1997, 20:71-80.

28. Baggs J: Collaborative interdisciplinary bioethics decision making in intensive care units. Nurs Outlook 1993, 41:108-112.

29. Baktoft B, Drigo E, Hohl ML, Klancar S, Tseroni M, Putzai P: A survey of critical care nursing education in Europe. Connect: World Crit Care Nurs 2008, 2:85-87.

30. Blackwood B, Alderdice F, Burns K, Cardwell C, Lavery G, O'Halloran P. Protocolized versus non-protocolized weaning for reducing the duration of mechanical ventilation in critically ill adult patients. Cochrane Database Syst Rev 2010, 5:CD006904.

31. ARDSnet: Ventilation with lower tidal volumes compared with traditional tidal volumes for acute lung injury and the acute respiratory distress syndrome. N Engl J Med 2000, 342:1301-1308.

32. Lellouche F, Mancebo J, Jolliet P, Roeseler J, Schortgen F, Dojat M, Cabello B, Bouadma L, Rodriguez P, Maggiore S, Reynaert M, Mersmann S, Brochard $L$ : A multicenter randomized trial of computer-driven protocolized weaning from mechanical ventilation. Am J Respir Crit Care Med 2006, 174:894-900.

33. Petter A, Chiolero R, Cassina T, Chassot PG, Muller XM, Revelly JP: Automatic respirator weaning with adaptive support ventilation: the effect on duration of endotracheal intubation and patient management. Anesth Analgesia 2003, 97:1743-1750.

34. Sulzer CF, Chiolero R, Chassot PG, Mueller XM, Revelly JP: Adaptive support ventilation for fast tracheal extubation after cardiac surgery: a randomized controlled study. Anesthesiol 2001, 95:1339-1345.

35. Dongelmans D, Veelo D, Paulus F, de Mol B, Korevaar J, Kudoga A, Middelhoek P, Binnekade J, Schultz M: Weaning automation with adaptive support ventilation: a randomized controlled trial in cardiothoracic surgery patients. Anesth Analg 2009, 108:565-571.

doi:10.1186/cc10588

Cite this article as: Rose et al: Decisional responsibility for mechanical ventilation and weaning: an international survey. Critical Care 2011 15:R295 